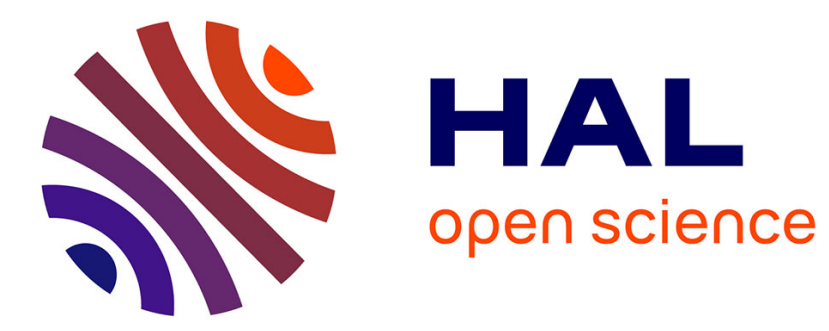

\title{
Assessing fire safety using complex numerical models with a Bayesian multi-fidelity approach
}

Rémi Stroh, Julien Bect, Séverine Demeyer, Nicolas Fischer, Damien Marquis, Emmanuel Vazquez

\section{- To cite this version:}

Rémi Stroh, Julien Bect, Séverine Demeyer, Nicolas Fischer, Damien Marquis, et al.. Assessing fire safety using complex numerical models with a Bayesian multi-fidelity approach. Fire Safety Journal, 2017, 91, pp.1016-1025. 10.1016/j.firesaf.2017.03.059 . hal-01568843v1

\section{HAL Id: hal-01568843}

\section{https://hal-centralesupelec.archives-ouvertes.fr/hal-01568843v1}

Submitted on 7 Aug 2017 (v1), last revised 20 Jan 2021 (v2)

HAL is a multi-disciplinary open access archive for the deposit and dissemination of scientific research documents, whether they are published or not. The documents may come from teaching and research institutions in France or abroad, or from public or private research centers.
L'archive ouverte pluridisciplinaire $\mathbf{H A L}$, est destinée au dépôt et à la diffusion de documents scientifiques de niveau recherche, publiés ou non, émanant des établissements d'enseignement et de recherche français ou étrangers, des laboratoires publics ou privés.

\section{(ㅇ)(1) $\$$}

Distributed under a Creative Commons Attribution - NonCommercial - NoDerivatives| 4.0 


\title{
Assessing Fire Safety using Complex Numerical Models with a Bayesian Multi-fidelity Approach
}

\author{
Rémi STROH ${ }^{1,3}$, Julien BECT ${ }^{3}$, Séverine DEMEYER ${ }^{1}$, Nicolas FISCHER ${ }^{1}$, Damien MARQUIS ${ }^{2}$ \\ and Emmanuel VAZQUEZ ${ }^{3}$ \\ ${ }^{1}$ Statistics and Mathematics Unit, Laboratoire National de métrologie et d'Essais (LNE) \\ 29, rue Roger Hennequin, 78190 Trappes; firstname.lastname@lne.fr \\ ${ }^{2}$ Fire Behavior and Safety Department, Laboratoire National de métrologie et d'Essais \\ ${ }^{3}$ Laboratoire des Signaux \& Systèmes (L2S), CentraleSupélec, Univ. Paris-Sud, Centre National de la \\ Recherche Scientifique (CNRS), Université Paris-Saclay \\ 3, Rue Joliot Curie, 91190 Gif-sur-Yvette; firstname.lastname@1ss.supelec.fr
}

\begin{abstract}
The use of accurate simulators based on Computational Fluid Dynamics (CFD) is becoming more and more common in fire safety analyses. However, the complexity of these numerical models drastically limits the number of simulations that can be afforded. Therefore, traditional methods of safety analysis are often difficult to apply. This paper proposes a statistical method to evaluate a quantity of interest with an expensive simulator while saving computation time. The method is based on Bayesian statistics and multi-fidelity. We use Gaussian process regression to construct a Bayesian model of the complex simulator. This model is based on a multi-fidelity approach, which consists in simulating at different levels of accuracy, for instance, by varying the spatial discretization in a CFD simulator. We illustrate our method on an example of fire safety analysis of a building, where the quantity of interest is the probability of exceeding a safety threshold.
\end{abstract}

KEYWORDS: risk assessment, statistics, numerical experiments, meta-model, multi-fidelity

\section{NOMENCLATURE LISTING}

\begin{tabular}{llll} 
General notations & $\left(z_{i}\right)$ & n values of observations \\
$f^{\text {env }}$ & distribution of environmental inputs & $n$ & computed with $n$ observations \\
$f_{x, t}^{\text {sim }}$ & distribution of output at $(x, t)$ & \multicolumn{2}{c}{ Estimation of conformity } \\
$\mathcal{N}$ & normal distribution & $n_{p}$ & number of sample paths \\
$t$ & fidelity input & $n_{x}$ & number of values of environmental inputs \\
$t_{H F}$ & highest level of fidelity & $P_{f}(\cdot)$ & probability of failure at $(\cdot)$ \\
$x$ & $=\left(x_{e}, x_{s}\right)$, physical inputs & $z^{\text {crit }}$ & critical safety threshold \\
$x_{e}$ & environmental inputs & Application \\
$x_{s}$ & scenario inputs & $A_{f}$ & fire area $\left(\mathrm{m}^{2}\right)$ \\
$Z$ & output of the simulator & $\alpha$ & fire growth acceleration $\left(\mathrm{kW} \cdot \mathrm{s}^{-2}\right)$ \\
Bayesian modeling & $H_{f}$ & smoke radiative heat flux $\left(\mathrm{kW} \cdot \mathrm{m}^{-2}\right)$ \\
$\mathrm{k}$ & covariance function & $P_{a t m}$ & atmospheric pressure $(\mathrm{bar})$ \\
$\lambda$ & variance of the stochastic effect & $\dot{Q}^{\prime \prime}$ & maximal heat release rate per unit area $\left(\mathrm{kW} \cdot \mathrm{m}^{-2}\right)$ \\
$\mathrm{m}$ & mean function & $q_{f}$ & total released energy per unit area $\left(\mathrm{MJ} \cdot \mathrm{m}^{-2}\right)$ \\
$\mu$ & value of a constant mean function & $T_{a m b}$ & ambient temperature $\left({ }^{\circ} \mathrm{C}\right)$ \\
$n$ & number of observations & $T_{c}$ & temperature at $1.8 \mathrm{~m}\left({ }^{\circ} \mathrm{C}\right)$ \\
$\left(x_{i}\right)$ & $n$ points of observations & $T_{e x t}$ & external temperature $\left({ }^{\circ} \mathrm{C}\right)$ \\
$\left(x_{i}, z_{i}\right)$ & $n$ observations & $V$ & visibility $(\mathrm{m})$ \\
$\xi$ & mean response of the simulator & $Y_{s o o t}$ & soot yield $\left(\mathrm{kg} \cdot \mathrm{kg}{ }^{-1}\right)$
\end{tabular}

\section{ACRONYMS}

CFD Computational Fluid Dynamics

DOE Design Of Experiments

FDS Fire Dynamics Simulator

GP Gaussian Process
HRR

LHS

OpenFOAM

STK
Heat Release Rate

Latin Hypercube Sampling

Open Field Operation And Manipulation

Small (Matlab/Octave) Toolbox for Kriging 


\section{INTRODUCTION}

The use of accurate simulators based on Computational Fluid Dynamics (CFD) is becoming more and more common in fire safety analyses. These simulators, such as Fire Dynamics Simulator (FDS) or Open Field Operation And Manipulation (OpenFOAM), can be used to test various fire scenarios, which would be too difficult or impossible to test using real experiments [1]. However, they are usually very time-consuming, which limits the number of numerical experiments that can be afforded. Consequently, traditional mathematical methods for fire safety analysis based on Monte-Carlo simulations [2; 3] become impractical, and fire safety engineers have to assess the safety of a building from a small number of simulation results. Moreover, operator dependence may cause different conclusions on a same problem, and complicate the comparison of experiments. In this context, there are various approaches which can help to carry out fire safety analyses using complex simulators.

This paper proposes a new approach, which is based on Bayesian statistics and multi-fidelity. Bayesian statistics is a mathematical framework for data analysis, where results are expressed in term of probability statements [4]. Each unknown quantity of interest is equipped with a probability distribution, which describes our knowledge of the quantity, and is updated whenever additional data becomes available (see [5] for an overview). The Bayesian method presented in this text is Gaussian process regression, which is used to model unknown complex simulators.

Multi-fidelity is an approach where the fast approximations of the simulator are available [6]. Typically, on a field model, such as FDS or OpenFOAM, the accuracy of the simulation can be reduced by increasing the spatial discretization. Theoretically, a simulation with coarser meshes describes a physical phenomenon with a low-fidelity, compared to a simulation with finer meshes. However, a low-fidelity simulation runs quicker than a high-fidelity simulation. The multi-fidelity approach uses low-fidelity simulations to learn more about high-fidelity [7]. We shall combine Bayesian statistics and multi-fidelity in order to estimate the fire predictions of a complex numerical model. This estimate will be used to assess the fire safety of a building and associate an uncertainty to the assessment.

In this paper, several uncertainties must be distinguished [8]. Thus, in our approach, we consider the simulator as a black-box, taking physical inputs $x$ and a fidelity input $t$, and returning a stochastic output $Z$ (see Figure 1). The fidelity input $t$ rules the level of the fidelity of simulations [9]. The physical input $x$ represent physical quantities (as temperatures, fire properties, boundary conditions...). Some of them, the environmental inputs, are unreliable or poorly known. The random of the environmental inputs is modeled by an input distribution (e.g., Gaussian or uniform distributions), which conveys a first uncertainty, called the input uncertainty. The output $Z$ (which may be the heat flux, the visibility...) is assumed stochastic. In fact, most CFD simulators are not stochastic, but, because of numerical details explained later in this paper, we can assume that the output is stochastic. At fixed inputs, the output follows a distribution $f_{x, t}^{\mathrm{sim}}(z)$, which represents the stochastic effect at $(x, t)$. The output distribution forms a second uncertainty, called the output uncertainty (at the fixed input).

This paper considers a third source of uncertainty, the Bayesian modeling uncertainty. In a Bayesian approach, each quantity is linked to a distribution. The uncertainty of this distribution represents the lack of knowledge of the quantity. Opposite to the previous two, this uncertainty can disappear when the number of simulations grows, because we learn more and more about the simulator, implying a reduction of its associated Bayesian modeling uncertainty.

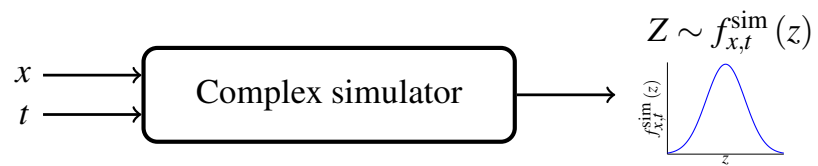

Fig. 1. Diagram of a numerical experiment. A simulator is described as a black-box. The implementation is ignored, only the inputs and output are considered. 
In this paper, we propose a statistical method to carry out analyses on a complex simulator, such as FDS. The presented method allows to evaluate any quantity of interest with minimal computation costs, and to associate an uncertainty, which is the uncertainty of the estimate. This final uncertainty depends on the three sources of uncertainty described above. We illustrate the methodology on conformity assessment of a building during a fire. The conformity is therefore described by a probability of exceeding a threshold, also called probability of failure (for instance, the probability that the heat flux exceeds $2.5 \mathrm{~kW} \cdot \mathrm{m}^{-2}$ ).

The paper is organized as follows. The first section will introduce Gaussian process regression. The second section will explain our methodology to estimate conformity thanks to Bayesian approach and multi-fidelity. And the third section will illustrate it with an example of fire safety in a building. The goal of the proposed methodology is to assess the safety of a building for a given fire scenario and associate an uncertainty on the assessment, with a complex numerical model such as FDS.

\section{BAYESIAN APPROACH TO MODELING THE OUTPUT OF THE SIMULATOR}

\section{Gaussian process regression}

The objective of this section is to construct a Bayesian model of the output of the simulator from the observations of a number of fire simulations. This Bayesian model will give a posterior probability distribution that will represent our knowledge about the simulator after the fire simulations. This posterior distribution of the simulator will then be used to assess the fire conformity of a building.

Let $\left(x_{i}, z_{i}\right)_{1 \leq i \leq n}$ be a dataset of $n$ observations of the simulator, where the $x_{i}$ 's are inputs, and the $z_{i}$ 's are the outputs associated to the $x_{i}$ 's. Each $x_{i}$ is a vector whose components correspond to the physical inputs of the simulator (temperatures, fire area...). Each $z_{i}$ corresponds to a scalar output of the simulation (e.g., the visibility) associated to $x_{i}$. In this section, the input corresponding to the mesh size is not considered for the sake of simplicity. However, there is no loss of generality in doing so. To model the fact that the output of the simulator is stochastic, we assume that the observed response is a random variable $Z$ with a Gaussian probability density function $f_{x}^{\text {sim }}$ such that

$f_{x}^{\operatorname{sim}}(z)=\frac{1}{\sqrt{2 \pi \lambda(x)}} \cdot e^{-\frac{1}{2} \cdot \frac{(z-\xi(x))^{2}}{\lambda(x)}}, \quad z \in \mathbb{R}$,

where $\xi(x)$ stands for the mean response of the simulator at $x$, and $\lambda(x)$ stands for the variance of the stochastic simulator at $x$. In this section, we assume that $\lambda$ is known, and $\xi$ is unknown.

To deal with the fact that $\xi$ is unknown, we assume that $\xi$ is a Gaussian Process (GP), which will be denoted by

$\xi \sim \mathcal{G P}(\mathrm{m}, \mathrm{k})$

where $\mathrm{m}$ and $\mathrm{k}$ are respectively the mean and covariance functions of $\xi$. The mean function is assumed to be constant and unknown. The covariance function is chosen by the user in such a way to describe how he/she expects two values $\xi(x)$ and $\xi\left(x^{\prime}\right)$ tend to be similar if $x$ and $x^{\prime}$ are close to each other. In particular, the covariance function specifies the smoothness of the simulator. From a Bayesian point of view, the Gaussian process represents our prior knowledge about the unknown mean response of the simulator.

Once our Bayesian model is set up, the next step is to condition the model on the observations $\left(x_{i}, z_{i}\right)_{1 \leq i \leq n}$. Bayes' theorem yields the posterior distribution of $\xi$. Under the assumptions of a constant mean function, $\mathrm{m}(x)=\mu$ and known covariance and variance functions $\mathrm{k}$ and $\lambda$, the posterior distribution of $\xi(x)$ is Gaussian, with mean

$\mathrm{m}_{n}(x)=\widehat{\mu}+\boldsymbol{k}^{T}(x)[K+\Lambda]^{-1}(\boldsymbol{z}-\widehat{\mu} \mathbf{1})$

and variance

$\sigma_{n}^{2}(x)=\mathrm{k}(x, x)-\boldsymbol{k}^{T}(x)[K+\Lambda]^{-1} \boldsymbol{k}(x)+\frac{\left(1-\mathbf{1}^{T}[K+\Lambda]^{-1} \boldsymbol{k}(x)\right)^{2}}{\mathbf{1}^{T}[K+\Lambda]^{-1} \mathbf{1}}$, 
where $\boldsymbol{z}=\left(z_{i}\right)$ are the outputs, $K=\left(\mathrm{k}\left(x_{i}, x_{j}\right)\right)$ is the observation covariance matrix, $\Lambda=\operatorname{Diag}\left(\lambda\left(x_{i}\right)\right)$ is the diagonal matrix of variances of stochastic effects at observation points, $\boldsymbol{k}(x)=\left(\mathrm{k}\left(x_{i}, x\right)\right)$ is the covariance vector between observations and the point $x, \mathbf{1}$ a $n \times 1$ is vector of ones, and $\widehat{\mu}=\left(\mathbf{1}^{T}[K+\Lambda]^{-1} \mathbf{1}\right)^{-1} \mathbf{1}^{T}[K+\Lambda]^{-1} z$ is the estimate of $\mu$. We refer the reader to Stein [10] or Forrester et al. [11] for more details about how these expressions can be established. Note that the function $\mathrm{m}_{n}$ can be seen as an estimator of $\xi$, and the function $\sigma_{n}$ can be seen as a measure of our lack of knowledge about $\xi$. The function $\mathrm{m}_{n}$ is also called a meta-model or an emulator of the simulator. The computation of the posterior distribution of $\xi$ is usually carried out using dedicated softwares. The Small (Matlab/Octave) Toolbox for Kriging (STK) [12] has been used in this paper. Once the posterior distribution is obtained, it can be used to estimate quantities of interest, and in particular, a probability of exceeding a safety threshold.

\section{Example}

This section illustrates the approach on a simple example. We consider a numerical model with a scalar input $x$, and returning a stochastic output $Z$. The quantity of interest is the mean response of the numerical model.

The first step of the Bayesian approach is to define a prior distribution. Although the numerical model is seen as a black-box, some assumptions can be made about it, for instance about its smoothness. These assumptions are translated into a Gaussian process $\xi$. Figure 2 a shows five sample paths of $\xi$, which represent what the mean response could be prior to any observation.

The second step is to observe the numerical model. Thus, $n$ inputs $x_{1}, \ldots, x_{n}$ are selected. Then, for each input $x_{i}$, the numerical model is run, and returns a result $z_{i}$. Figure $2 \mathrm{~b}$ shows $n=20$ observations $\left(x_{i}, z_{i}\right)$ corresponding to the stochastic outcome of the numerical model.

The conditioning of the prior distribution produces the posterior distribution of the output of the numerical model. Figure $2 \mathrm{c}$ shows five sample paths of the posterior distribution. The sample paths of the posterior distribution resemble those of the prior distribution, but they now "capture" the observations.

Finally, Figure $2 \mathrm{~d}$ represents an estimate of the unknown mean response. The solid line is the function $\mathrm{m}_{n}$ (Eq. (3)), which is an estimator of the unknown mean response. The inner area around the line is a $95 \%$ confidence interval of the estimate, which is proportional to $\sigma_{n}$ (Eq. (4)). The outer area is a $95 \%$ confidence interval of future observations, taking into account the stochastic effect. In other words, a new observation of the unknown numerical model has a $95 \%$ chance to be in this area. Confidence intervals are sometimes called credible intervals by Bayesian statisticians.

\section{PROPOSED METHODOLOGY}

\section{Multi-fidelity}

Because of the complexity of CFD simulators, the number of observations that can be made is limited. In this section, we will detail how, by making specific assumptions about the Bayesian model presented above, we can derive a multi-fidelity model.

Multi-fidelity is a solution to reduce the uncertainty in the posterior distribution at a small budget of computation time. In a simulator based on a field model, such as FDS or OpenFOAM, the accuracy of a simulation is controlled by the spatial discretization. A high-density mesh improves space accuracy (hence should also increase fidelity to physical reality) but also increases computation time. The multi-fidelity approach consists in simulating at different levels of fidelity, instead of only the highest one. Thus, the idea is to combine fast low-fidelity simulations (by changing the mesh size) with slow high-fidelity simulations to improve the quality of the posterior model.

In the literature, multi-fidelity often deals with only two levels of fidelity [6;13]. In this article, we work with a continuum of levels of fidelity indexed by a positive scalar $t$, following Picheny and Ginsbourger [9] and Tuo et al. [14]. The level $t$ can be seen as an input of the simulator, as the input vector $x$ (see Figure 1). In this work, since the level of fidelity $t$ is related to the mesh size, fidelity increases when $t$ tends to zero, even if this level of spatial discretization is impossible to simulate. We denote by $t_{H F}$ the highest available level of fidelity 


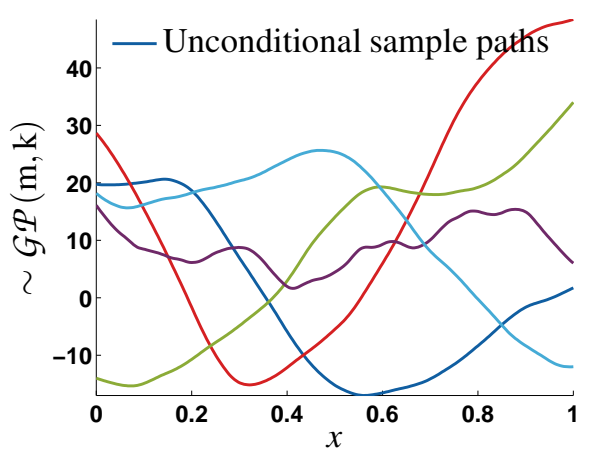

(a) Sample paths of the prior distribution

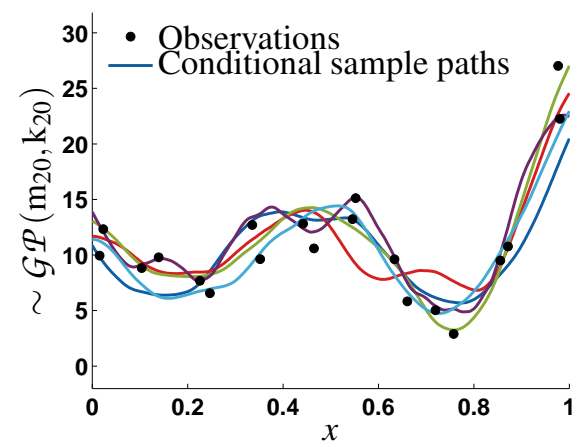

(c) Sample paths of the posterior distribution

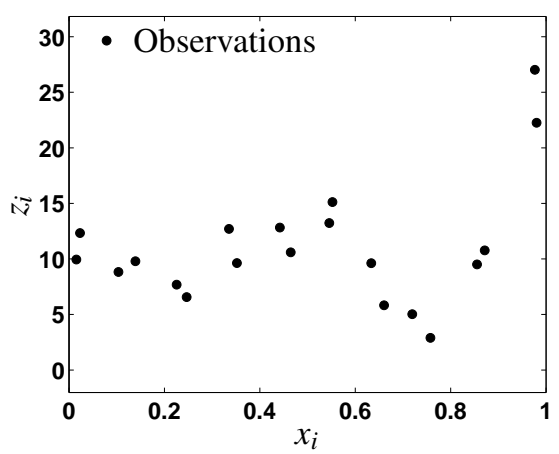

(b) Observations of an unknown function $(n=20)$

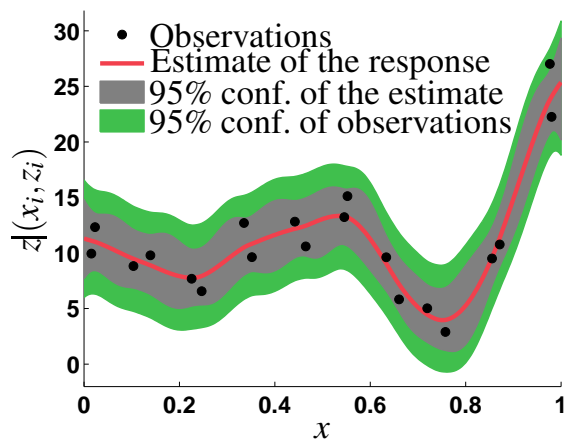

(d) Evaluation with the posterior distribution

Fig. 2. Illustration of Gaussian process regression. (a) The unconditioned Gaussian process. (b) Observations of the numerical model. (c) The conditioned Gaussian process. (d) An estimate of the mean response.

(lowest value of $t$, corresponding to the finest meshes). This ideal level of fidelity $t_{H F}$ should be selected in order to adequately resolve the fluid flow and fire dynamics. In the case of a CFD code, where $t$ corresponds to the size of mesh cells, Lin et al. [15] recommends to use $t_{H F} \leq D^{\star} / 10$, with $D^{\star}$ the characteristic fire diameter.

We develop a Gaussian process of the global simulator, valid for any level of spatial discretization. The first subsection proposes a prior multi-fidelity model of the simulator. Then, the second subsection describes how the numerical experiments are selected. Conditioning the prior distribution by the numerical observations creates the posterior distribution, which can evaluate any quantity of interest. Thus, the third subsection shows an example of evaluation, with an estimation of probability of failure. Note that, even if we focus only on the highest level of fidelity $t_{H F}$, this posterior model could be used at any level of fidelity, in other word, for each spatial resolution.

\section{Multi-fidelity prior model}

The simulators that we aim to describe have a fidelity input $t$ and a stochastic output $Z$. Following the model of stochastic simulator described Eq. (1), we suppose that the output $Z$ at $(x, t)$ follows a normal probability distribution, whose parameters depend on $(x, t)$ :

$f_{x, t}^{\operatorname{sim}}(z)=\frac{1}{\sqrt{2 \pi \lambda(x, t)}} \cdot e^{-\frac{1}{2} \cdot \frac{(z-\xi(x, t))^{2}}{\lambda(x, t)}}$,

where $\xi(x, t)$ and $\lambda(x, t)$ are respectively the mean and the variance of the output at $(x, t)$, which are both assumed unknown now. The next paragraphs present briefly our prior models about $\xi$ and $\lambda$. 
The prior distribution of the mean response $\xi$ was proposed by Tuo et al. [14] for deterministic simulators. To build the prior model, the mean response $\xi$ is assumed to be the sum of an ideal response $\xi_{0}$ and a numerical error $\varepsilon$ :

$\xi(x, t)=\xi_{0}(x)+\varepsilon(x, t)$,

where the ideal response $\xi_{0}$ corresponds to the case where $t$ tends to zero, whereas the numerical error $\varepsilon$ stems from the numerical approximations of the equations of physics. Thus, we assume that $\varepsilon(x, t=0)=0$ for all $x$. We suggest taking a covariance function proposed by Tuo et al. [14]:

$\mathrm{k}\left((x, t),\left(x^{\prime}, t^{\prime}\right)\right)=\mathrm{k}_{0}\left(x, x^{\prime}\right)+\min \left\{t, t^{\prime}\right\}^{L} \mathrm{k}_{\varepsilon}\left(x, x^{\prime}\right)$,

where $\mathrm{k}_{0}$ is the covariance function of the ideal response $\xi_{0}$ and $\mathrm{k}_{\varepsilon}$ that of the numerical error $\varepsilon$. The term $\min \left\{t, t^{\prime}\right\}^{L}$ is such that that the numerical error decreases when $t$ decreases, i.e., when fidelity increases. Notice that the numerical error and the stochastic effect are two distinct parts of the simulator. The simulator would still be stochastic, even if the numerical error was zero.

The prior distribution on the noise variance $\lambda$ is described in Stroh et al. [16]. In order to simplify the model, we suppose that the noise variance depends only on the levels of spatial discretization, so that $\lambda$ depends only on the fidelity levels $t$. Then, the noise variance is assumed to be different at each level, but have the same order of magnitude. These assumptions are summed up in a log-normal prior distribution.

\section{Design of Experiments}

This section presents how numerical experiments are selected once the Bayesian model has been set.

In our multi-fidelity framework, we choose the set of observations, also called Design Of Experiments (DOE), in such a way that the computation time remains low. It is well-known that to construct a good Bayesian model, the DOE should be space-filling (see Pronzato and Müller [17] for a review). One of the most popular method to obtain a space-filling DOE is to use maximin Latin Hypercube Sampling (LHS). A design is LHS, when the inputs are regularly spaced along all one-dimensional axis (see Figure $3 \mathrm{a}$ for an example of space-filling LHS). More details can be found in Santner et al. [18].

In our multi-fidelity approach, since low-fidelity is cheaper than high-fidelity, low-fidelity should be more often observed than high-fidelity. To this purpose, we use nested LHS. A design is said to be nested, when each point observed at an high-fidelity level is also observed at lower-fidelity levels (see Figure 3b).

In order to construct a nested LHS design, we use the algorithm developed by Qian [19], which produces nested LHS design, with an additional maximin optimization at each level, to obtain a good space-filling.

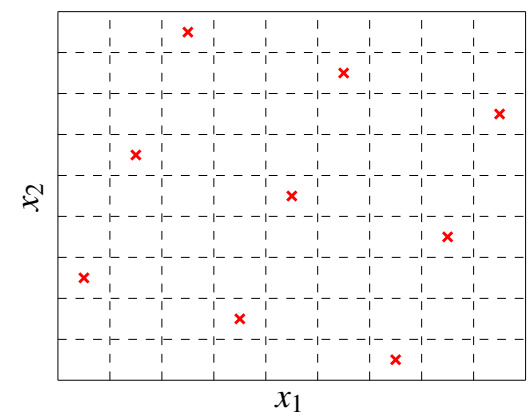

(a) A space-filling LHS, $n=9, d=2$

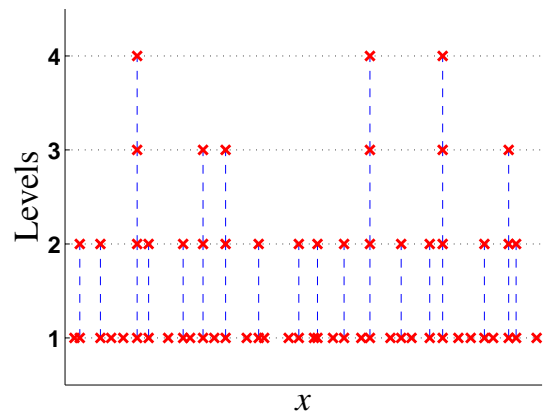

(b) A nested design, $n=36+18+6+3, d=1, S=4$

Fig. 3. Two examples of design. (a) A Latin Hypercube Sampling. (b) A nested design (the lowest level of fidelity is below). $n$ is the number of points, $d$ the dimension, $S$ the number of levels of fidelity. 


\section{Estimation of the conformity of a building using the multi-fidelity model}

Given the results of the simulations, we can compute the posterior distribution which can be used in turn to estimate quantities of interest, and therefore assess the conformity of a building during a fire. In this paper, conformity is assessed by comparing the output $Z$ to a critical threshold $z^{\text {crit }}$. More precisely, we estimate the probability that $Z$ exceeds the threshold $z^{\text {crit }}$ in various fire scenarios, in order to find which scenarios are the most dangerous.

The procedure to estimate the probability of failure at a given fire scenario is based on two steps. First, we consider a pointwise probability of failure defined as the probability that the output $Z$ at $(x, t)$ exceeds its safety threshold $z^{\text {crit }}$ (for instance, the probability that the heat flux predicted by the simulator at a given fire scenario, weather conditions and spatial discretization exceeds $2.5 \mathrm{~kW} \cdot \mathrm{m}^{-2}$ ),

$P_{f}(x, t)=\mathbb{P}\left(Z>z^{\text {crit }}\right)=\int_{z^{\text {crit }}}^{+\infty} f_{x, t}^{\text {sim }}(z) \mathrm{d} z$,

where $f_{x, t}^{\operatorname{sim}}(z)$ denotes the output density at $(x, t)$ (see Introduction). Recall from (5) that $Z$ is assumed to follow a normal distribution. Given $\xi$ and $\lambda$, the pointwise probability of failure can be computed as

$P_{f}(x, t)=\Phi\left(\frac{\xi(x, t)-z^{\text {crit }}}{\sqrt{\lambda(x, t)}}\right)$,

where $\Phi$ is the normal cumulative distribution function.

Until here, all components of the input $x$ have been treated without distinction. Now, two kinds of inputs are distinguished: the environmental inputs $x_{e}$ on the one hand, and the fire scenario inputs $x_{s}$ on the other hand (in such a way that $x=\left(x_{e}, x_{s}\right)$ ). The environmental inputs can vary in time, which is modeled by a distribution $f^{\text {env }}\left(x_{e}\right)$ representing the variations of the environmental inputs. We would like to express the probability of failure only as a function of the scenario inputs $x_{s}$, to assess their impact on the conformity of the building during a fire. Of course, our objective is to estimate the conformity at high-fidelity level, so that the fidelity parameter is fixed to $t_{H F}$. Then, the probability of failure can be written as

$P_{f}\left(x_{s}\right)=\int P_{f}\left(x_{s}, x_{e}, t_{H F}\right) f^{\text {env }}\left(x_{e}\right) \mathrm{d} x_{e}$.

This second type of probability of failure depends on the output and input distributions. Since, $P_{f}\left(x_{s}\right)$ depends on $\xi$ and $\lambda, P_{f}\left(x_{s}\right)$ has a posterior distribution which depends on those of $\xi$ and $\lambda$.

The posterior distribution of $P_{f}\left(x_{s}\right)$ has no closed-form expression. Consequently, numerical approximations must be used. We resort to a Monte-Carlo method. To estimate the probability of failure given a scenario $x_{s}$, we begin by drawing randomly $n_{x}$ values of environmental inputs $\left(x_{e, i}\right)$ from the distribution $f^{\text {env }}$. Then, we draw randomly $n_{p}$ sample paths of $\xi$ and $\lambda$ from the posterior distribution (see Figure $2 \mathrm{c}$ ). For each path, the probability of failure can be estimated by computing the pointwise probability of failure, and averaging on the environmental input samples:

$p_{j}\left(x_{s}\right)=\frac{1}{n_{x}} \sum_{i=1}^{n_{x}} P_{f}^{(j)}\left(x_{e, i}, x_{s}, t_{H F}\right), \quad 1 \leq j \leq n_{p}$

The $p_{j}$ 's are approximate samples of the posterior distribution of $P_{f}\left(x_{s}\right)$.

From these samples, a point estimate and an uncertainty can be computed. We use respectively median and $95 \%$ confidence interval. The uncertainty of the estimate takes account on the uncertainty of the environmental inputs (when $n_{x}$ values of environmental inputs are generated), of the output (when pointwise probability of failure are computed), and of the posterior Bayesian model (when $n_{p}$ sample paths are drawn from posterior distribution). This algorithm has to be applied for each scenario of interest.

\section{Summary of the methodology}

Consider a fire safety study which has to be effected on a numerical field model. The numerical model is a stochastic complex simulator, taking physical inputs $x$ and a fidelity input $t$, and returning an output $Z$. The 
inputs $x$ must be the most significant on the output. Other parameters must be set, and are supposed known and constant. The simulations should ideally be run at the level of fidelity $t_{H F}$. The inputs $x$ are divided in two categories: environmental inputs $x_{e}$ and scenario inputs $x_{s}, x=\left(x_{e}, x_{s}\right)$. A distribution $f^{\text {env }}\left(x_{e}\right)$ on environmental inputs is specified. In order to estimate any quantity of interest, the procedure consists of:

1. Define different levels of fidelity, which must be lower fidelity than $t_{H F}$. Generate a nested Latin Hypercube Sampling design $\left(x_{i}, t_{i}\right)_{1 \leq i \leq n}$ according to the computation budget. For each point $\left(x_{i}, t_{i}\right)$ of the design, run the corresponding simulation, and get the output $z_{i}$.

2. Condition the Gaussian process which models the simulator to the observations $\left(x_{i}, t_{i}, z_{i}\right)_{1 \leq i \leq n}$.

3. Compute the posterior distribution of the quantity of interest. Deduce an estimate and an uncertainty. For instance, the method described above can be used to estimate a probability of failure.

\section{APPLICATION}

\section{Description of the case}

To illustrate the methodology, we present an example of fire safety analysis. We consider a real building of the Laboratoire National de métrologie et d'Essais (LNE), which is a $20 \mathrm{~m}$ by $12 \mathrm{~m}$ by $16 \mathrm{~m}$ rectangular parallelepiped. The thickness and the thermal properties of the walls are defined in accordance with the present structure. It has two open doors at the floor level, $2 \mathrm{~m}$ by $1 \mathrm{~m}$, and two natural smoke removal systems in the ceiling, $2 \mathrm{~m}$ by $2 \mathrm{~m}$ (Figures $4 \mathrm{a}$ and $4 \mathrm{~b}$ ). The fire source is located in the center of the test hall, and is described by a Heat Release Rate (HRR) evolution in three steps which are growing, steady state and decreasing (Figure 4c). Concerning the boundary conditions, the gas in the computational domain is set still with ambient temperature, and, at the free sides, a static pressure boundary condition is employed.

The conformity of the smoke control system is estimated by comparing tenability conditions to critical thresholds (see Table 1). The three studied outputs are the radiative heat flux $H_{f}$, the temperature at $1.8 \mathrm{~m} T_{c}$, and the visibility $V$. A simulation of FDS returns these three outputs. During a simulation, sensors are put in the whole building at $1.8 \mathrm{~m}$ above the ground, except above the plume of the fire (see Figure $4 \mathrm{~b}$ ). The distance between the sensors is $1 \mathrm{~m}$. Each sensor returns a time evolution of the three outputs. A post-treatment transforms these time evolutions into one scalar value per output. The values on all sensors are averaged at each time step, and then the extremal value of this time-dependent average is computed (maximum for the heat flux and the temperature, minimum for the visibility). The outputs are analyzed separately.

The study is carried out using FDS version 5.5.3.. It is a CFD model of fire-driven fluid flow, developed by the National Institute of Standards and Technology (NIST), which solves an approximation of the Navier-Stokes

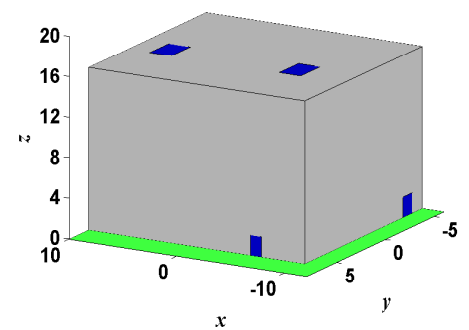

(a) External view

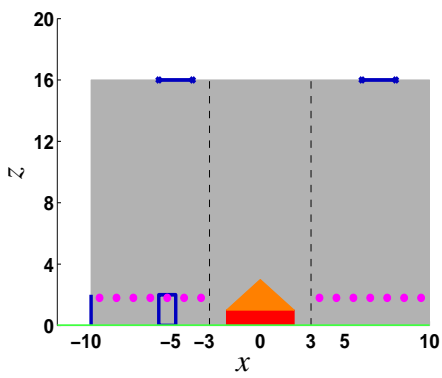

(b) Section at $y=0$

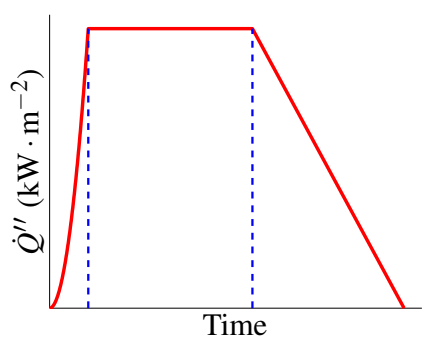

(c) Sample of a design fire curve

Fig. 4. (a) External view of the considered building. (b) Section of the building. The triangle in the middle is the fire, the bullet points are the sensors. (c) Time evolution of the Heat Release Rate. 


\begin{tabular}{|lr|l|l|}
\hline \multicolumn{2}{|c|}{ Outputs } & \multicolumn{1}{|c|}{ Signification } & \multicolumn{1}{|c|}{ Failure } \\
\hline$H_{F}$ & $\left(\mathrm{~kW} \cdot \mathrm{m}^{-2}\right)$ & Radiative Heat Flux & $>2.5 \mathrm{~kW} \cdot \mathrm{m}^{-2}$ \\
$T_{c}$ & $\left({ }^{\circ} \mathrm{C}\right)$ & Temperature at $1.8 \mathrm{~m}$ & $>60{ }^{\circ} \mathrm{C}$ \\
$V$ & $(\mathrm{~m})$ & Visibility & $<5 \mathrm{~m}$ \\
\hline
\end{tabular}

Table 1. List of outputs studied during this experiment.

equations appropriate for low-Mach number using finite difference methods [20]. Strictly speaking, FDS is a deterministic simulator. Indeed, random fields are initialized with a pseudo-random number generator, but whose seed is fixed. However, the outputs of FDS are very irregular functions of the inputs, similarly to those of a stochastic simulator, which is why we choose to model FDS, from a statistical point of view, as a stochastic simulator.

We focus on eight particular input variables, which are the most influential ones. Three of them are considered as environmental inputs (external temperature, atmospheric pressure, and ambient temperature), while the five other are categorized as scenario inputs (fire growth acceleration, fire area, maximal surface power, total surface energy, and soot yield). Moreover, the size $t_{x y z}$ of the mesh cells is used as the fidelity input in our multi-fidelity approach. See Table 2 for more information.

Numerical experiments are performed according to a nested LHS with four levels of observations: 270 simulations at level $100 \mathrm{~cm}, 90$ at $50 \mathrm{~cm}, 30$ at $33 \mathrm{~cm}, 10$ at $25 \mathrm{~cm}$ and none at $20 \mathrm{~cm}$, which correspond to a total computation time of approximately 21.4 days (see Table 3). The highest level of fidelity is $t_{H F}=20 \mathrm{~cm}$.

\section{Results}

This section presents results obtained with our methodology. First, as an example of the kind of results that can be obtained from the posterior distribution. Figure 5 presents the estimates of the three outputs along the fire area $A_{f}$ axis, at high-fidelity level $t_{x y z}=20 \mathrm{~cm}$ (other inputs being fixed at $T_{\text {ext }}=10^{\circ} \mathrm{C}, P_{a t m}=1 \mathrm{bar}$, $T_{a m b}=22.5^{\circ} \mathrm{C}, \alpha=0.1057 \mathrm{~kW} \cdot \mathrm{s}^{-2}, \dot{Q}^{\prime \prime}=460 \mathrm{~kW} \cdot \mathrm{m}^{-2}, q_{f_{d}}=450 \mathrm{MJ} \cdot \mathrm{m}^{-2}$ and $\left.Y_{\text {soot }}=0.027 \mathrm{~kg} \cdot \mathrm{kg}^{-1}\right)$. The solid line is the mean function of the posterior distribution, which is our estimator of $\xi$ (defined in Eq. (1)). The inner area around the line is the $95 \%$ confidence interval of the estimate. The outer area is the $95 \%$ confidence interval of future simulations, which also takes into account the output variability measured by the variance $\lambda$ (defined in Eq. (1)). We can observe that, as expected, the building becomes more dangerous when the fire area grows.

Let us now consider the evaluation of conformity for some fire scenarios. As written in previous sections, the building conformity can be assessed by the probability of exceeding the safety threshold (Table 1). We let the fire area $A_{f}$ vary between $1 \mathrm{~m}^{2}$ and $20 \mathrm{~m}^{2}$, with one scenario every $0.25 \mathrm{~m}^{2}$. Other scenario inputs are fixed to $\alpha=0.1057 \mathrm{~kW} \cdot \mathrm{s}^{-2} ; \dot{Q}^{\prime \prime}=460 \mathrm{~kW} \cdot \mathrm{m}^{-2} ; q_{f_{d}}=450 \mathrm{MJ} \cdot \mathrm{m}^{-2} ; Y_{\text {soot }}=0.027 \mathrm{~kg} \cdot \mathrm{kg}^{-1}$. Thus, there are 77 fire scenarios for which the probability of failure is estimated. We use the method described in the previous section to estimate probability of exceeding the threshold, with $n_{p}=10^{6}$ sample paths and $n_{x}=700$ values of environmental inputs. Estimates are presented in Figure 6. The solid line is the estimate of the probability of exceeding the threshold at the given scenario. The area is the $95 \%$ confidence interval. Once again, we find that the place becomes more dangerous when fire becomes bigger. These graphs show also the critical scenario of the building. Thus, other scenario inputs being fixed, the building is safe until the fire area $A_{f}$ is greater than $12 \mathrm{~m}^{2}$. We can also observe on the visibility, Figure $6 \mathrm{c}$, that estimation of probability of failure becomes very inaccurate around $17 \mathrm{~m}^{2}$. In fact, the width of the confidence interval becomes equal to 1 , implying that nothing can be deduced from the data about the value of the probability $P_{f}$ at this point. To fix this, additional simulations must be added to reduce the uncertainty. Of course, the inputs of these simulations should be properly selected to provide as much information as possible. The methods for selecting the inputs to observe are called sequential design of experiments.

In this subsection, the presented results depend on the fire area $A_{f}$. However, the results (distribution of outputs, probability of failure...) can be analyzed according to any inputs $\left(\dot{Q}^{\prime \prime}, \alpha \ldots\right.$, or many of them). 


\begin{tabular}{|c|c|c|c|}
\hline \multicolumn{2}{|c|}{ Random inputs } & Signification & Distributions \\
\hline & $\left({ }^{\circ} \mathrm{C}\right)$ & External temperature & $T_{\text {ext }}\left({ }^{\circ} \mathrm{C}\right) \sim \mathcal{N}\left(10,6.66^{2}\right)$ \\
\hline$P_{a t m}$ & (bar) & Mean atmospheric pressure & $P_{\text {atm }}($ bar $) \sim \mathcal{N}\left(1,0.00666^{2}\right)$ \\
\hline$T_{a m b}$ & $\left({ }^{\circ} \mathrm{C}\right)$ & Initial ambient temperature & $T_{a m b}\left({ }^{\circ} \mathrm{C}\right) \mid T_{\text {ext }}\left({ }^{\circ} \mathrm{C}\right) \sim \mathcal{N}\left(22.5+2 \frac{\left(T_{e x t}-10\right)}{6.66}, 1.5^{2}\right)$ \\
\hline \multicolumn{2}{|c|}{ Scenario inputs } & Signification & $\overline{\text { Ranges }}$ \\
\hline & $\left(\mathrm{kW} \cdot \mathrm{s}^{-2}\right)$ & Growth acceleration & {$[0.011338 ; 0.2] \mathrm{kW} \cdot \mathrm{s}^{-2}$} \\
\hline & $\left(\mathrm{m}^{2}\right)$ & Fire area & {$[1 ; 20] \mathrm{m}^{2}$} \\
\hline$\dot{Q}^{\prime \prime}$ & $\left(\mathrm{kW} \cdot \mathrm{m}^{-2}\right)$ & Maximal Heat Release Rate & {$[300 ; 600] \mathrm{kW} \cdot \mathrm{m}^{-2}$} \\
\hline & $\left(\mathrm{MJ} \cdot \mathrm{m}^{-2}\right)$ & Total surface released energy & {$[300 ; 600] \mathrm{MJ} \cdot \mathrm{m}^{-2}$} \\
\hline$Y_{\text {Soot }}$ & $\left(\mathrm{kg} \cdot \mathrm{kg}^{-1}\right)$ & Soot yield & {$[0.01227 ; 0.1227] \mathrm{kg} \cdot \mathrm{kg}^{-1}$} \\
\hline \multicolumn{2}{|c|}{ Fidelity input } & Signification & Values \\
\hline$\overline{t_{x y z}}$ & $(\mathrm{~cm})$ & Mesh size & $\{100 ; 50 ; 33 ; 25 ; 20\} \mathrm{cm}$ \\
\hline
\end{tabular}

Table 2. List of inputs studied during this experiment. $\mathcal{N}$ is the normal distribution.

\begin{tabular}{lccccc|c}
\hline Levels & $100 \mathrm{~cm}$ & $50 \mathrm{~cm}$ & $33 \mathrm{~cm}$ & $25 \mathrm{~cm}$ & $20 \mathrm{~cm}$ & Total \\
\hline Number of simulations in the design & 270 & 90 & 30 & 10 & $\mathbf{0}$ & 400 \\
\hline Approximative computation time & $4.7 \mathrm{~min}$ & $65 \mathrm{~min}$ & $6.2 \mathrm{~h}$ & $21 \mathrm{~h}$ & 2.3 days & 21.4 days \\
Comparison with level $20 \mathrm{~cm}$ & $1 / 700$ & $1 / 50$ & $1 / 8.8$ & $1 / 2.6$ & 1 & 9.5 \\
\hline
\end{tabular}

Table 3. Description of the levels. Remarks: the computation time depends on the hardware, and the simulations can be parallelized.

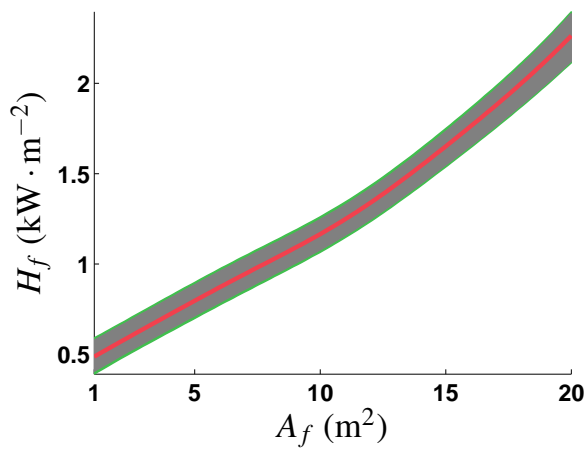

(a) Heat Flux

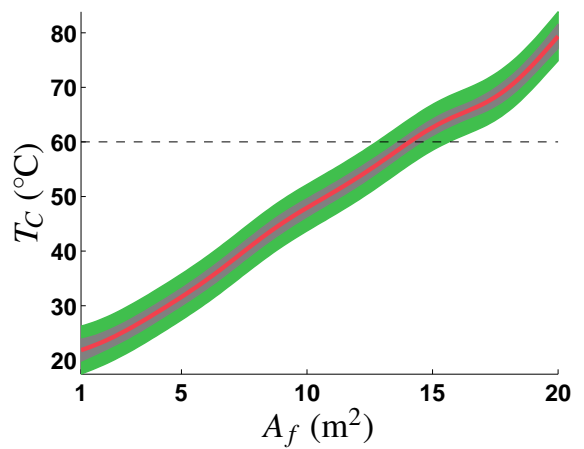

(b) Temperature

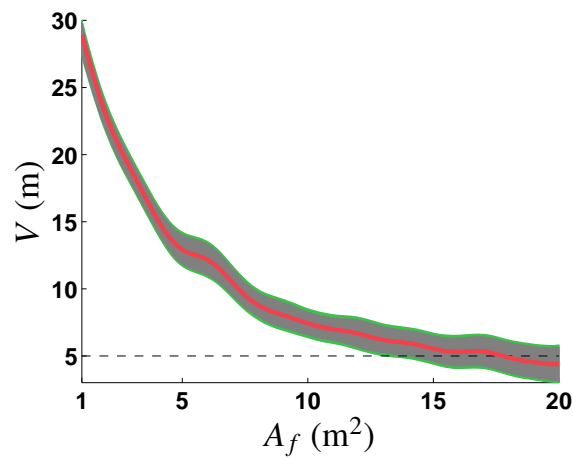

(c) Visibility

Fig. 5. Estimates of the three outputs as function of the fire area $A_{f} \in[1 ; 20] \mathrm{m}^{2}$. (a) Heat Flux $H_{f}$. (b) Temperature $T_{c}$. (c) Visibility $V$. The safety threshold of $H_{f}$ is never reached. 


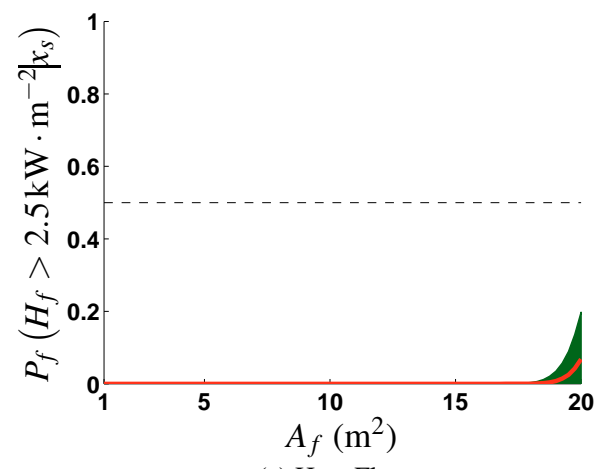

(a) Heat Flux

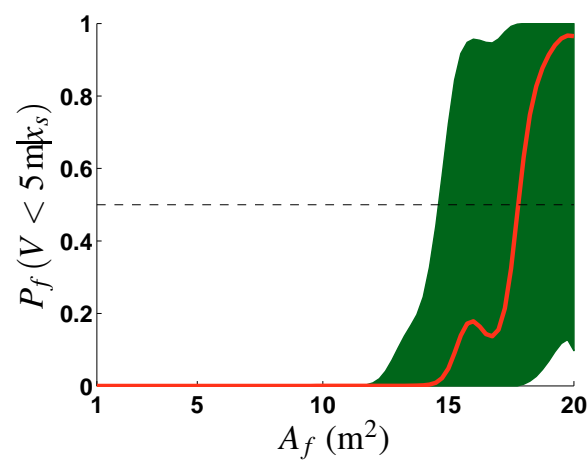

(c) Visibility

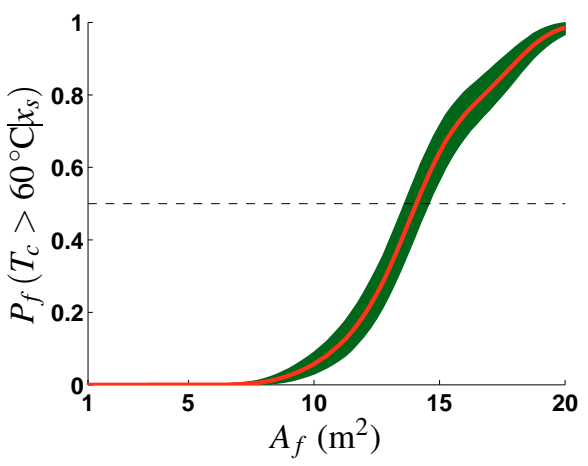

(b) Temperature
- Estimates of the probability of failure 95\% confidence interval

Fig. 6. Estimates of probabilities of failure as function of the scenario variable $A_{f} \in[1 ; 20] \mathrm{m}^{2}$, the fire area. Each figure corresponds to one output: (a) Heat Flux $H_{f}$, (b) Temperature $T_{c}$ and (c) Visibility $V$.

The Bayesian approach provides an estimate and a corresponding measure of uncertainty for any quantity of interest.

The relevance of our method can be seen by comparing computation time (see Table 3 ). In fact, we can observe that our multi-level design takes $270 \times \frac{4.7}{60 \times 24}+90 \times \frac{65}{60 \times 24}+30 \times \frac{6.2}{24}+10 \times \frac{21}{24}=21.4$ days to be evaluated. In such a delay, about $\frac{21.4}{2.3}=9.5$ high-fidelity simulations could be run, which is too few to draw any conclusion about output values at unobserved locations or probability of failure.

\section{Validation on additional data}

The previous part presented the estimates obtained with a Bayesian multi-fidelity approach. This part compares estimates with outputs of high-fidelity simulations, to assess their accuracy. In fact, there is no simulation on the highest level of fidelity (see Table 3). However, we use our method to study a non-observed level. Consequently, we must ensure that the method can estimate a non-observed level.

To do this, we launch 100 new simulations at $20 \mathrm{~cm}$, the highest fidelity level. These new data are designed according to an optimized maximin LHS, but are not involved in the construction of the conditional Gaussian processes. The previously built posterior distribution estimates the outputs at the inputs of these high-fidelity simulations. Thus, estimates (with uncertainty) can be compared with high-fidelity simulations.

Figure 7 presents the comparison between simulations and estimates at the highest level of fidelity, for the three outputs. On these figures, estimates and simulations are distributed around the $y=x$ line. The estimates seems good on the whole input space. Observe that a few uncertainty bars do not cross the $y=x$ line, which is expected since they are $95 \%$ confidence intervals. 


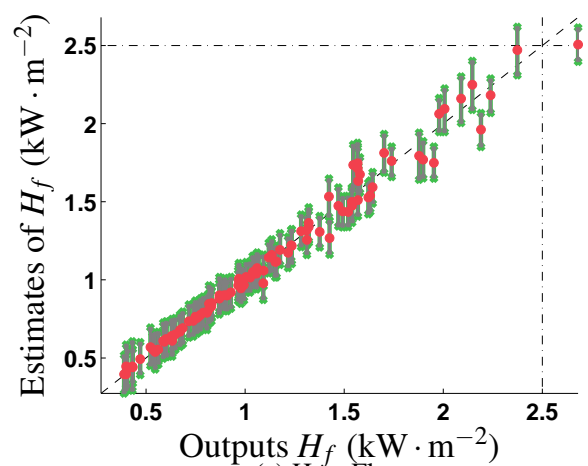

(a) Heat Flux

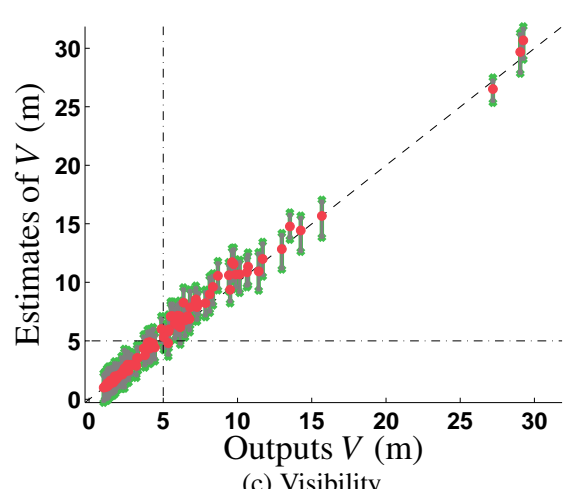

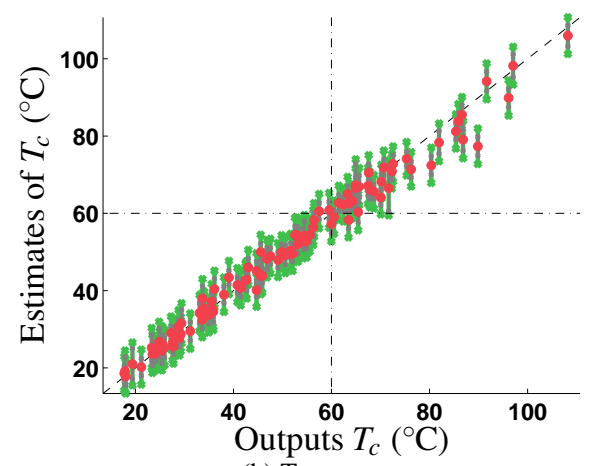

(b) Temperature
- Estimates of the outputs

$-95 \%$ confidence interval

$\rightarrow$ Stochastic effect

-... Safety threshold

Fig. 7. Comparison between estimates (ordinate) and high-fidelity simulations (abscissa), for the three outputs: (a) Heat Flux $H_{f}$, (b) Temperature $T_{c}$ and (c) Visibility $V$. The dashed diagonals are the lines $y=x$.

The method can also be checked by comparing the estimate of the probability of failure with the estimate obtained with another method. To do it, the following fire scenario is chosen: $\alpha=0.1057 \mathrm{~kW} \cdot \mathrm{s}^{-2}, A_{f}=$ $14 \mathrm{~m}^{2}, \dot{Q}^{\prime \prime}=460 \mathrm{~kW} \cdot \mathrm{m}^{-2}, q_{f_{d}}=450 \mathrm{MJ} \cdot \mathrm{m}^{-2}, Y_{\text {soot }}=0.027 \mathrm{~kg} \cdot \mathrm{kg}^{-1}$. This fire scenario is present on the curves presented Figure 6 . The results obtained by our method for this specific scenario are compared with estimates of the probabilities of failure by Monte-Carlo, using 150 high-fidelity simulations.

Figure 8 presents the different results returned by both methods. For each figure, the left result is obtained with our methodology and the uncertainty is measured as a $95 \%$ confidence interval, and the right one is computed with Monte-Carlo and the uncertainty is measured as a 95\% confidence interval. For the smoke radiative Heat Flux, both methods estimate a probability of failure at 0 . For the Temperature, both results are similar. For the Visibility, our methodology returns a larger 95\% interval than the Monte-Carlo method. As emphasized earlier, some additional simulations are needed to learn on the Visibility. In the three cases, the results of both methods are compatible.

Recall that the results presented for our methodology are based on a dataset which took 21.4 days to be computed. In contrast, the design used by Monte-Carlo method takes $150 \times 2.3=345$ days (see Table 3 ). So, our method is $\frac{345}{21.4}=16$ time faster than a direct Monte-Carlo method. Moreover, the Monte-Carlo method gives a probability of failure for one scenario, while our methodology can provide estimates of the probability of failure for any scenario. 


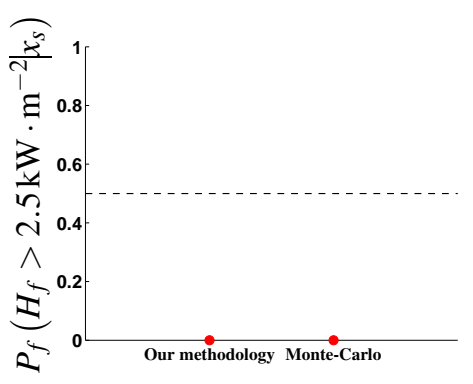

(a) Heat Flux

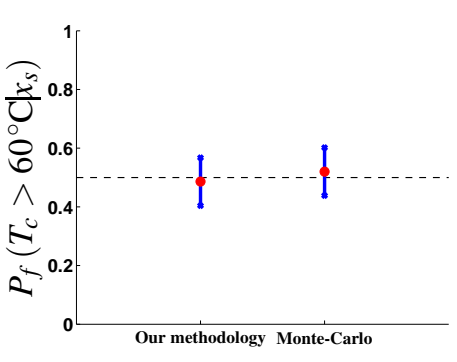

(b) Temperature

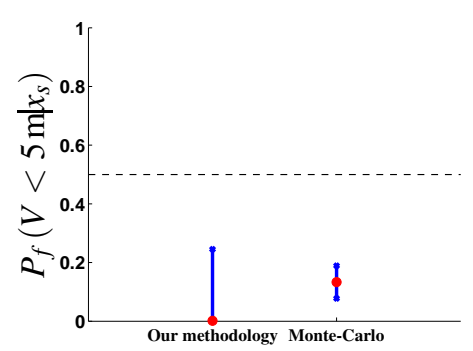

(c) Visibility

Fig. 8. Comparison between the estimates of probabilities of failure returned by the method (left on figures) and by Monte-Carlo (right on figures). The bullet points are estimates, and the bars are $95 \%$ uncertainties.

\section{CONCLUSION}

This article provides a method to assess the fire safety of a building using a complex numerical model, such as FDS or OpenFOAM. The proposed method is based on a Bayesian multi-fidelity approach. More exactly, we use a Gaussian process model to estimate the complex simulator with minimal computation cost. The prior Gaussian model, which represents our assumption about the simulator, and observations of the simulator are combined to construct a posterior distribution, which is used to estimate quantities of interest, such as, in this paper, the probability of exceeding a safety threshold.

Because of the complexity of the CFD model, the number of observation is limited. The multi-fidelity approach is a way to reduce the uncertainties of estimates for a given budget computation time. The idea consists in combining fast low-fidelity and slow high-fidelity simulations of the CFD simulator to construct the posterior distribution.

The methodology was tested on a fire safety study. By comparing the computation time, the Bayesian multifidelity approach was found to be ten times faster than a standard one-level approach, on this example. The developed Bayesian models can estimate the expected outputs of the simulator at the highest level of fidelity, and probabilities of failure at given fire scenarios. Moreover, the posterior distributions provide a quantification of uncertainty for the quantities of interest. Our methodology assesses safety quicker and with more confidence than with traditional analyses.

However, the methodology can be improved. The prior distribution is constructed on various assumptions, and some of them are strong. Checking the validity of the Bayesian model will improve the confidence on results. The methodology can also be improved when simulations are selected. The DOE which we proposed is based following the general recommendations of the statistical community. However, the observations of the numerical model can be selected for the specific purpose of improving the estimation of a quantity of interest. Future work will focus on Bayesian model validation and sequential design of experiments in multifidelity.

\section{REFERENCES}

[1] Pablo Ayala, Alexis Cantizano, Guillermo Rein, Gabriele Vigne, and Cándido. Gutiérrez-Montes. Fire experiments and simulations in a full-scale atrium under transient and asymmetric venting conditions. Fire Technology, 52(1):51-78, 2016. doi: 10.1007/s10694-015-0487-9.

[2] Siu Kui Au, Zhi-Hua Wang, and Siu-Ming Lo. Compartment fire risk analysis by advanced monte carlo simulation. Engineering Structures, 29(9):2381-2390, 2007. doi: http://dx.doi.org/10.1016/j. engstruct.2006.11.024.

[3] Depeng Kong, Nils Johansson, Patrick van Hees, Shouxiang Lu, and Siuming Lo. A Monte Carlo analysis of the effect of heat release rate uncertainty on available safe egress time. Journal of Fire Protection Engineering, 23(1):5-29, 2012. doi: 10.1177/1042391512452676. 
[4] Kevin McGrattan and Blaza Toman. Quantifying the predictive uncertainty of complex numerical models. Metrologia, 48(3):173, 2011.

[5] Andrew Gelman, John B. Carlin, Hal S. Stern, David B. Dunson, Aki Vehtari, and Donald B. Rubin. Bayesian data analysis. Chapman \& Hall/CRC, third edition, 2013.

[6] Marc C. Kennedy and Anthony O'Hagan. Predicting the output from a complex computer code when fast approximations are available. Biometrika, 87(1):1-13, 2000. doi: 10.1093/biomet/87.1.1.

[7] Loic Le Gratiet and Claire Cannamela. Cokriging-based sequential design strategies using fast cross-validation techniques for multi-fidelity computer codes. Technometrics, 57(3):418-427, 2015. doi: 10.1080/00401706.2014.928233.

[8] Marc C. Kennedy and Anthony O’Hagan. Bayesian calibration of computer models. Journal of the Royal Statistical Society: Series B (Statistical Methodology), 63(3):425-464, 2001. doi: 10.1111/1467-9868.00294.

[9] Victor Picheny and David Ginsbourger. A nonstationary space-time gaussian process model for partially converged simulations. SIAM/ASA Journal on Uncertainty Quantification, 1(1):57-78, 2013. doi: $10.1137 / 120882834$.

[10] Michael L. Stein. Interpolation of Spatial Data: Some Theory for Kriging. Springer Series in Statistics. Springer, New York, 1999. doi: 10.1007/978-1-4612-1494-6.

[11] Alexander I. J. Forrester, András Sóbester, and Andy J. Keane. Engineering Design via Surrogate Modelling: A Practical Guide. Wiley, 2008.

[12] Julien Bect, Emmanuel Vazquez, et al. STK: a Small (Matlab/Octave) Toolbox for Kriging. Release 2.3., 2014.

[13] Peter Z. G. Qian and C. F. Jeff Wu. Bayesian hierarchical modeling for integrating lowaccuracy and high-accuracy experiments. Technometrics, 50(2):192-204, 2008. doi: 10.1198/ 004017008000000082 .

[14] Rui Tuo, C. F. Jeff Wu, and Dan Yu. Surrogate modeling of computer experiments with different mesh densities. Technometrics, 56(3):372-380, 2014. doi: 10.1080/00401706.2013.842935.

[15] Chih-Hung Lin, Yuh-Ming Ferng, and Wen-Shieng Hsu. Investigating the effect of computational grid sizes on the predicted characteristics of thermal radiation for a fire. Applied Thermal Engineering, 29(11-12):2243-2250, 2009. doi: http://dx.doi.org/10.1016/j.applthermaleng.2008.11.010.

[16] Rémi Stroh, Julien Bect, Séverine Demeyer, Nicolas Fischer, and Emmanuel Vazquez. Gaussian process modeling for stochastic multi-fidelity simulators, with application to fire safety. In 48èmes Journées de Statistique de la SFdS (JdS 2016), Montpellier, France, may 2016.

[17] Luc Pronzato and Werner G. Müller. Design of computer experiments: space filling and beyond. Statistics and Computing, 22(3):681-701, 2012. doi: 10.1007/s11222-011-9242-3.

[18] Thomas J. Santner, Brian J. Williams, and William I. Notz. The Design and Analysis of Computer Experiments. Springer Series in Statistics. Springer, New York, 2003. doi: 10.1007/ 978-1-4757-3799-8.

[19] Peter Z. G. Qian. Nested latin hypercube designs. Biometrika, 96(4):957-970, 2009. doi: 10.1093/ biomet/asp045.

[20] Kevin McGrattan, Simo Hostikka, and Jason E. Floyd. Fire dynamics simulator (version 5), user's guide. National Institute of Standards and Technology (NIST), 2010. 